\title{
Persistence and baseline determinants of seropositivity and reinfection rates in health care workers up to 12.5 months after COVID-19
}

Carlota Dobaño ( $\sim$ carlota.dobano@isglobal.org)

ISGlobal, Hospital Clínic - Universitat de Barcelona, Barcelona https://orcid.org/0000-0002-6751-4060 Anna Ramirez

IDIAP Jordi Gol https://orcid.org/0000-0001-8263-1292

\section{Selena Alonso}

ISGlobal, Hospital Clínic - Universitat de Barcelona, Barcelona https://orcid.org/0000-0003-4029-9716

Josep Vidal-Alaball

Institut Català de la Salut https://orcid.org/0000-0002-3527-4242

\section{Gemma Ruiz-Olalla}

ISGlobal

\section{Marta Vidal}

ISGlobal, Hospital Clínic - Universitat de Barcelona, Barcelona

\section{Rocio Rubio}

ISGlobal

\section{Emma Cascant}

ISGlobal

\section{Daniel Parras}

IDIBAPS https://orcid.org/0000-0002-2677-4245

Natalia Rodrigo Melero

CRG

Pau Serra

IDIBAPS

\section{Carlo Carolis}

Centre for Genomic Regulation (CRG), The Barcelona Institute of Science and Technology, Barcelona https://orcid.org/0000-0003-4240-1139

\section{Pere Santamaria}

Faculty of Medicine, University of Calgary

\section{Anna Forcada}

Gerència territorial de Catalunya Central

Jacobo Mendioroz

ICS 


\section{Ruth Aguilar}

ISGlobal, Hospital Clínic - Universitat de Barcelona, Barcelona https://orcid.org/0000-0003-3277-3107 Gemma Moncunill

ISGlobal, Hospital Clínic - Universitat de Barcelona, Barcelona https://orcid.org/0000-0001-5105-9836 Anna Ruiz-Comellas

IDIAP Jordi Gol

\section{Brief Communication}

Keywords: SARS-CoV-2, COVID-19, antibodies, spike antigen, duration, kinetics, reinfection, health care workers, cohort, baseline determinants

Posted Date: May 14th, 2021

DOI: https://doi.org/10.21203/rs.3.rs-142984/v2

License: (c) (1) This work is licensed under a Creative Commons Attribution 4.0 International License. Read Full License 


\section{Abstract}

We assessed the duration and baseline determinants of antibody responses to SARS-CoV-2 spike antigens and the occurrence of reinfections in a prospective cohort of 173 Spanish primary health care worker patients followed up initially for nine months and subsequently up to 12.5 months after COVID-19 symptoms onset. Seropositivity to SARS-CoV-2 spike and receptor binding domain antigens up to 149270 days was $92.49 \%(90.17 \% \lg , 76.3 \% \lg A, 60.69 \% \lg M)$. In a subset of 64 health care workers who had not yet been vaccinated by April 2021 , seropositivity was $96.88 \%(95.31 \% \lg$ G, $82.81 \% \lg A)$ up to $322-$ 379 days post symptoms onset. There were four suspected reinfections detected by passive case detection, two among seronegative individuals (five and seven months after the first episode), and one low antibody responder. Antibody levels significantly correlated with fever, hospitalization, anosmia/hypogeusia, allergies, smoking and occupation. Stable sustainment of IgG responses raises hope for long-lasting COVID-19 vaccine immunity.

\section{Introduction}

A key question to understand the evolution of the COVID-19 pandemic is the duration of immune response generated to SARS-CoV-2. Most patients induce a robust humoral and cellular response [1] but with high heterogeneity and a percentage of non-responders. Diversity in epitope specificity, quality and functional capacity of antibodies will likely affect the efficacy of the immunity mediated. Antibodies elicited after exposure to SARS-CoV-2 have been associated with protective immunity up to 6 months [25], although we do not yet have a correlate of protection, and reinfections occur seemingly at a low frequency. The spike (S) protein on the virus surface is considered the main target of protective antibodies, and the component of the leading vaccines [6] already under implementation. Functional neutralizing antibodies highly correlate with IgG levels to the receptor binding domain (RBD) of S [1], but $\lg \mathrm{A}$ and $\lg \mathrm{M}$ also have neutralizing properties [7].

Despite an increasing understanding of the nature of antibody responses, their longevity remains to be defined as the pandemic evolves. The duration of protective antibodies is a critical question as reinfection rates may increase if immunity wanes. Although initial reports indicated a decline in antibodies after 3 months [8], subsequent studies have shown relatively stable antibody levels, mostly $\lg \mathrm{G}$, over a period of up to 6 months and beyond [1-5, 9-17]. As massive global immunization campaigns advance, this knowledge will give clues as to how long COVID-19 vaccine immunity might last and how preexisting SARS-CoV-2 antibodies and other baseline variables could affect vaccine effectiveness.

\section{Methods}

\section{Study subjects}


Demographic and clinical data were collected to characterize the factors associated with disease presentation, presence of sequelae, long COVID-19 and reinfection in a cohort of 173 primary health care workers (HCW) in Barcelona, Spain, recruited at the first peak of the pandemic (March-April 2020). Baseline symptoms recorded included fever, shivers, headache, asthenia, myalgia, arthralgia, dyspnea, chest pain, cough, sputum production, hemoptysis, anosmia, hypoageusia, odynophagia, tachycardia, dizziness and thrombosis. For the multivariable regression analysis, symptoms were grouped into categories: digestive, otolaryngology, neurological, ophthalmology, and skin disorders. Baseline information collected included also history of previous allergies and smoking habits.

Five cross-sectional surveys were done between September to November 2020, and January to April 2021, to obtain venous blood for assessing maintenance of anti-SARS-CoV-2 seropositivity and analyze baseline factors associated with antibody levels. Participants were not systematically monitored for potential asymptomatic reinfections. Vaccinated HCW were excluded from this analysis.

The study protocol was approved by the IRB Comitè Ėtic d'Investigació Clínica IDIAP Jordi Gol (codes 20/094-PCV and 20/162-PCV) and written informed consent was obtained from participants.

\section{Laboratory analyses}

Levels of $\lg M$, IgA and $\lg G$ to RBD and $S$ recombinant proteins expressed from plasmids donated by $F$. Krammer (Mount Sinai, NY) were quantified in plasma by Luminex, as described [18]. The cutoff for seropositivity was calculated with 128 prepandemic samples as 10 to the mean plus 3 standard deviations of $\log _{10}$-transformed mean fluorescence intensity values.

\section{Data analysis}

Antibody levels were correlated with days since onset of symptoms, and results expressed in Spearman coefficient (rho) and $p$ values. Univariable and multivariable stepwise linear regression models were fit to determine the effect of baseline variables on the antibody levels $\left(\log _{10}\right)$ in the full cohort before the start of vaccination (December 2020). Models were selected based on the Akaike information criterion, Bayesian information criterion and adjusted r-square parameters. A transformed beta value (\%) of the loglinear model was calculated with the formula: ((10^beta)-1)*100, giving the difference (in percentage) in antibody levels when comparing to the reference group for categorical variables or for a one-unit change for continuous variables, for easier interpretation of the beta value results. All p-values were considered statistically significant when $<0.05$. All data collected were managed and analyzed using the $\mathrm{R}$ software version 3.6.3.

\section{Results And Discussion}

Most clinical cases in this cohort of HCW were mild-moderate COVID-19, with 24 hospitalized, and 64 presenting with sequelae. Median age was 49 years (IQR 41-58), 137 were females, and there were 13 smokers and 31 ex-smokers. 
We did not detect a significant decline in antibody levels as a function of time since symptoms onset (Figure 1). The percentage of seropositivity 149-270 days after symptoms onset combining RBD and S antigens was $60.69 \%$ for $\lg \mathrm{M}, 76.30 \%$ for $\lg \mathrm{A}$, and $90.17 \%$ for $\lg$, consistent with the expected longer duration of the latter isotype. Unexpectedly, seropositivity was quite sustained also for IgM and IgA, considered to be isotypes of shorter duration. Computing all immunoglobulins, seroprevalence 5-9 months after the initial COVID-19 episode was as high as $92.49 \%$, indicating very stable persistence of responses. Furthermore, 64 of $173 \mathrm{HCW}$ not yet vaccinated were tested in January and April 2021, and the overall percentage of seropositivity up to 322-379 days after onset of symptoms in this subset was still as high as $96.88 \%$ (IgG $95.31 \%$, IgA $82.81 \%$, IgM 25.0\%). These $64 \mathrm{HCW}$ had a seropositivity of $98.44 \%$ at 5-9 months after the initial COVID-19 (IgG 95.31\%, IgA $87.50 \%$, IgM $37.50 \%)$.

There were four suspected reinfections (Table 1). Before the second positive polymerase chain reaction (PCR) diagnosis, two symptomatic cases were seronegative, one asymptomatic was seropositive with low antibodies, and one had unknown serostatus. We attempted to recover the viral RNA from the first episodes for genome sequencing and demonstration of different strains but unfortunately it was not stored. This data set provides some indication of the frequency of reinfection in 173 primary infections with three likely reinfections (interval $>90$ days as per CDC guidelines) and one suspected reinfection ( $<90$ days between primary and reinfection). Therefore, there was a minimal overall rate of symptomatic reinfection of 2/173 (1.16\%). This rate contrasts with what we found in another HCW cohort that we followed up for 7-month seroprevalence in which no reinfections were detected $[19,5]$. It could be that primary HCW are more at risk of reinfection than hospital-based HCW, although it should be pointed out that is based on limited sample size. The study also provides some evidence that a lack of $S$ antibody response is a risk factor for symptomatic reinfection while positive serology leads to asymptomatic reinfection (Table 1). This is relevant due to the strong correlation (rho $=0.9)$ between antibody levels to $S$ and RBD with neutralizing function that are thought to confer protection [5].

Stepwise multivariable regression analyses showed that the baseline factors most consistently and significantly associated with higher levels of antibodies 5-9 months after infection were having been admitted to hospital, presenting fever $(n=131)$, anosmia and/or hypogeusia $(n=106)$, and having had previous allergies ( $n=24)$ (Table 2). Specifically, for anti-S IgG, HCW with fever had 2.5 times higher levels, patients with anosmia and/or hypogeusia had 2.6 times higher levels, and those with allergies had 1.9 times higher levels, than patients without those conditions. Baseline factors associated with lower levels of IgA and IgG included being a nurse $(n=68)$ or a physician $(n=70)$ compared to other occupation categories working in primary health care centers including customer and social services staff $(n=35)$, and smoking. For anti-S IgA, physicians had $34.84 \%$ and nurses $45.67 \%$ lower levels than the other jobs, and smokers had $46.17 \%$ less than non-smokers (Table 2). Nurses included eight auxiliary nurses, and physicians included one dentist. Other factors were associated with only certain isotypes. Presenting with sputum and/or hemoptysis $(n=13)$ was associated with higher IgM levels, and shivers $(n=86)$ were associated with higher IgAs. Of note, hospitalized patients had 2.1 times higher IgM levels to RBD than non-hospitalized. Age correlated positively with IgGs, having $1.39 \%$ higher antibody levels to RBD with each year of age older (Table 2). Higher IgGs (and IgAs less strongly) positively correlated with duration 
of symptoms (median 24 days, IQR 13-36; $S$ rho=0.229 $P=0.002 ; R B D$ rho=0.246, $P=0.001$ ) and number of symptoms (median 10, IQR 6-12; S rho $=0.351 \mathrm{P}<0.001$; RBD rho $=0.364, \mathrm{P}<0.001$ ). All other variables, symptoms, or sequelae, were either not statistically significantly associated with antibody levels or weakly in univariable models.

Previous acute phase studies showed that COVID-19 severity was associated with higher antibody responses. Here, hospitalization was associated with higher immunoglobulin levels many months after convalescence, suggesting that severity does not affect stability of memory B cell and plasma cells producing antibodies $[2-4,20]$. Common symptoms like fever and highly specific symptoms like alteration in smell and taste were also associated with higher antibodies. Interestingly, having previous allergies also correlated positively with higher antibody levels, which to our knowledge has not been reported. This could be related to disease exacerbation and increased risk of respiratory infections associated with some allergies [21] although this relationship remains unclear. Lower antibody levels in nurses and physicians than other HCW could indicate a lower exposure due to PPE use and higher awareness of risks [19]. Smoking had previously been associated with lower antibody responses [22, 23] and we show that this effect persists after several months primarily affecting $\lg A$, the main mucosal antibody.

In conclusion, despite the large heterogeneity in antibody levels induced by SARS-CoV-2 infection, most HCW patients remained seropositive for anti-S antibodies up to 12.5 months after COVID-19. The findings that after PCR reversion, 2 out of 13 seronegatives had another symptomatic episode, and that one low responder had a second (asymptomatic) infection, are consistent with a protective role for antibodies [24]. Considering that antibody levels achieved by COVID-19 immunization are usually higher than those elicited following natural infection, based on this study it could be speculated that immune memory induced by first generation vaccines could also be long-lasting, therefore reducing the probability that periodical boosters might be required to sustain protective immunity, at least within the first year. Furthermore, data indicates that naïve people should be prioritized for vaccination over those who had suffered COVID-19 since the latter maintain antibodies for at least a year.

\section{Declarations}

\section{ACKNOWLEDGEMENTS}

We thank the HCW for their participation in the study. We are grateful to Chenjerai Jairoce and Robert Mitchell for assistance with sample processing.

The study received support from the Fundació Privada Daniel Bravo Andreu. Development of SARS-CoV-2 reagents was partially supported by the NIAID Centers of Excellence for Influenza Research and Surveillance (CEIRS) contract HHSN272201400008C. ISGlobal receives support from the Spanish Ministry of Science and Innovation through the "Centro de Excelencia Severo Ochoa 2019-2023" Program (CEX2018-000806-S), and support from the Generalitat de Catalunya through the CERCA Program. 


\section{AUTHOR CONTRIBUTIONS}

Designed the study: CD, GM, JVA, ARC, ARM. Recruited and followed up patients and collected clinical data: ARM, JVA, AF, JM, ARC. Performed the laboratory analysis: SA, RR, MV. Performed the statistical analysis: GRO, SA. Produced the proteins for immunoassays: DP, NRM, PS, CC, PS. Coordinated or managed the study and/or laboratory work: EC, RA, GM, CD. Wrote the first draft: CD; Revised and approved the manuscript: all.

\section{Conflict of interest statement}

Authors declare no conflict of interest

\section{References}

1. Wajnberg A, Amanat F, Firpo A, Altman DR, Bailey MJ, Mansour M, et al. Robust neutralizing antibodies to SARS-CoV-2 infection persist for months. Science (80- ). 2020;370:1227-30.

2. Abayasingam A, Balachandran $H$, Agapiou D, Hammoud M, Rodrigo $C$, Keoshkerian $E$, et al. Longterm persistence of RBD+ memory B cells encoding neutralizing antibodies in SARS-CoV-2 infection. Cell Reports Med. 2021;2:100228.

3. Sokal A, Chappert P, Barba-Spaeth G, Roeser A, Fourati S, Azzaoui I, et al. Maturation and persistence of the anti-SARS-CoV-2 memory B cell response. Cell. 2021;184:1201-1213.e14.

4. Sherina N, Piralla A, Du L, Wan H, Kumagai-Braesch M, Andréll J, et al. Persistence of SARS-CoV-2specific $B$ and T cell responses in convalescent COVID-19 patients 6-8 months after the infection. Med. 2021;2:281-295.e4.

5. Ortega N, Ribes M, Vidal M, Rubio R, Aguilar R, Williams S, et al. Seven-month kinetics of SARS-CoV-2 antibodies and protective role of pre-existing antibodies to seasonal human coronaviruses on COVID19. medRxiv. 2021;:2021.02.22.21252150. doi:10.1101/2021.02.22.21252150.

6. Krammer F. SARS-CoV-2 vaccines in development. Nature. 2020;586:516-27. doi:10.1038/s41586020-2798-3.

7. Klingler J, Weiss S, Itri V, Liu X, Oguntuyo KY, Stevens C, et al. Role of IgM and IgA Antibodies in the Neutralization of SARS-CoV-2. medRxiv. 2020;:2020.08.18.20177303.

8. Seow J, Graham C, Merrick B, Acors S, Pickering S, Steel KJA, et al. Longitudinal observation and decline of neutralizing antibody responses in the three months following SARS-CoV-2 infection in humans. Nat Microbiol. 2020;5:1598-607. doi:10.1038/s41564-020-00813-8.

9. Dan JM, Mateus J, Kato Y, Hastie KM, Yu ED, Faliti CE, et al. Immunological memory to SARS-CoV-2 assessed for up to eight months after infection. Science (80- ). 2021;371:eabf4063.

10. Choe PG, Kim KH, Kang CK, Suh HJ, Kang EK, Lee SY, et al. Antibody responses 8 months after asymptomatic or mild SARS-CoV-2 infection. Emerg Infect Dis. 2021;27:928-31.

doi:10.3201/eid2703.204543. 
11. Whitcombe AL, McGregor R, Craigie A, James A, Charlewood R, Lorenz N, et al. Comprehensive analysis of SARS-CoV-2 antibody dynamics in New Zealand. medRxiv. 2020;:2020.12.10.20246751.

12. Figueiredo-Campos P, Blankenhaus B, Mota C, Gomes A, Serrano M, Ariotti S, et al. Seroprevalence of anti-SARS-CoV-2 antibodies in COVID-19 patients and healthy volunteers up to 6 months post disease onset. Eur J Immunol. 2020;50:2025-40. doi:10.1002/eji.202048970.

13. Ripperger TJ, Uhrlaub JL, Watanabe M, Wong R, Castaneda Y, Pizzato HA, et al. Orthogonal SARSCoV-2 Serological Assays Enable Surveillance of Low-Prevalence Communities and Reveal Durable Humoral Immunity. Immunity. 2020;53:925-933.e4.

14. Liu C, Yu X, Gao C, Zhang L, Zhai H, Hu Y, et al. Characterization of antibody responses to SARS-CoV2 in convalescent COVID-19 patients. J Med Virol. 2021;93:2227-33. doi:10.1002/jmv.26646.

15. Gaebler C, Wang Z, Lorenzi JCC, Muecksch F, Finkin S, Tokuyama M, et al. Evolution of Antibody Immunity to SARS-CoV-2. bioRxiv. 2020;:2020.11.03.367391.

16. Pradenas E, Trinité B, Urrea V, Marfil S, Ávila-Nieto C, de la Concepción MLR, et al. Stable neutralizing antibody levels six months after mild and severe COVID-19 episode. bioRxiv. 2020;:2020.11.22.389056.

17. Chen Y, Zuiani A, Fischinger S, Mullur J, Atyeo C, Travers M, et al. Quick COVID-19 Healers Sustain Anti-SARS-CoV-2 Antibody Production. Cell. 2020.

18. Dobaño $C$, Vidal M, Santano R, Jiménez A, Chi J, Barrios D, et al. Highly sensitive and specific multiplex antibody assays to quantify immunoglobulins $M, A$ and $G$ against SARS-CoV-2 antigens. J Clin Microbiol. 2020;59:e01731-20.

19. Moncunill G, Mayor A, Santano R, Jiménez A, Vidal M, Tortajada M, et al. SARS-CoV-2 Seroprevalence and Antibody Kinetics among Health Care Workers in a Spanish Hospital after 3 Months of Followup. J Infect Dis. 2021;223:62-71. doi:10.1093/infdis/jiaa696.

20. Hartley GE, Edwards ESJ, Aui PM, Varese N, Stojanovic S, McMahon J, et al. Rapid generation of durable B cell memory to SARS-CoV-2 spike and nucleocapsid proteins in COVID-19 and convalescence. Sci Immunol. 2020;5. doi:10.1126/sciimmunol.abf8891.

21. Yang JM, Koh HY, Moon SY, Yoo IK, Ha EK, You S, et al. Allergic disorders and susceptibility to and severity of COVID-19: A nationwide cohort study. J Allergy Clin Immunol. 2020;146:790-8. doi:10.1016/j.jaci.2020.08.008.

22. Schaffner A, Risch L, Aeschbacher S, Risch C, Weber MC, Thiel SL, et al. Characterization of a PanImmunoglobulin Assay Quantifying Antibodies Directed against the Receptor Binding Domain of the SARS-CoV-2 S1-Subunit of the Spike Protein: A Population-Based Study. J Clin Med. 2020;9:3989. doi:10.3390/jcm9123989.

23. Jonsdottir HR, Bielecki M, Siegrist D, Buehrer TW, Züst R, Deuel JW. Titers of Neutralizing Antibodies against SARS-CoV-2 Are Independent of Symptoms of Non-Severe COVID-19 in Young Adults. Viruses. 2021;13. doi:10.3390/v13020284.

24. Lumley SF, O'Donnell D, Stoesser NE, Matthews PC, Howarth A, Hatch SB, et al. Antibody Status and Incidence of SARS-CoV-2 Infection in Health Care Workers. N Engl J Med. 2020. 


\section{Tables}

Table 1. Characteristics of the suspected SARS-CoV-2 reinfection cases.

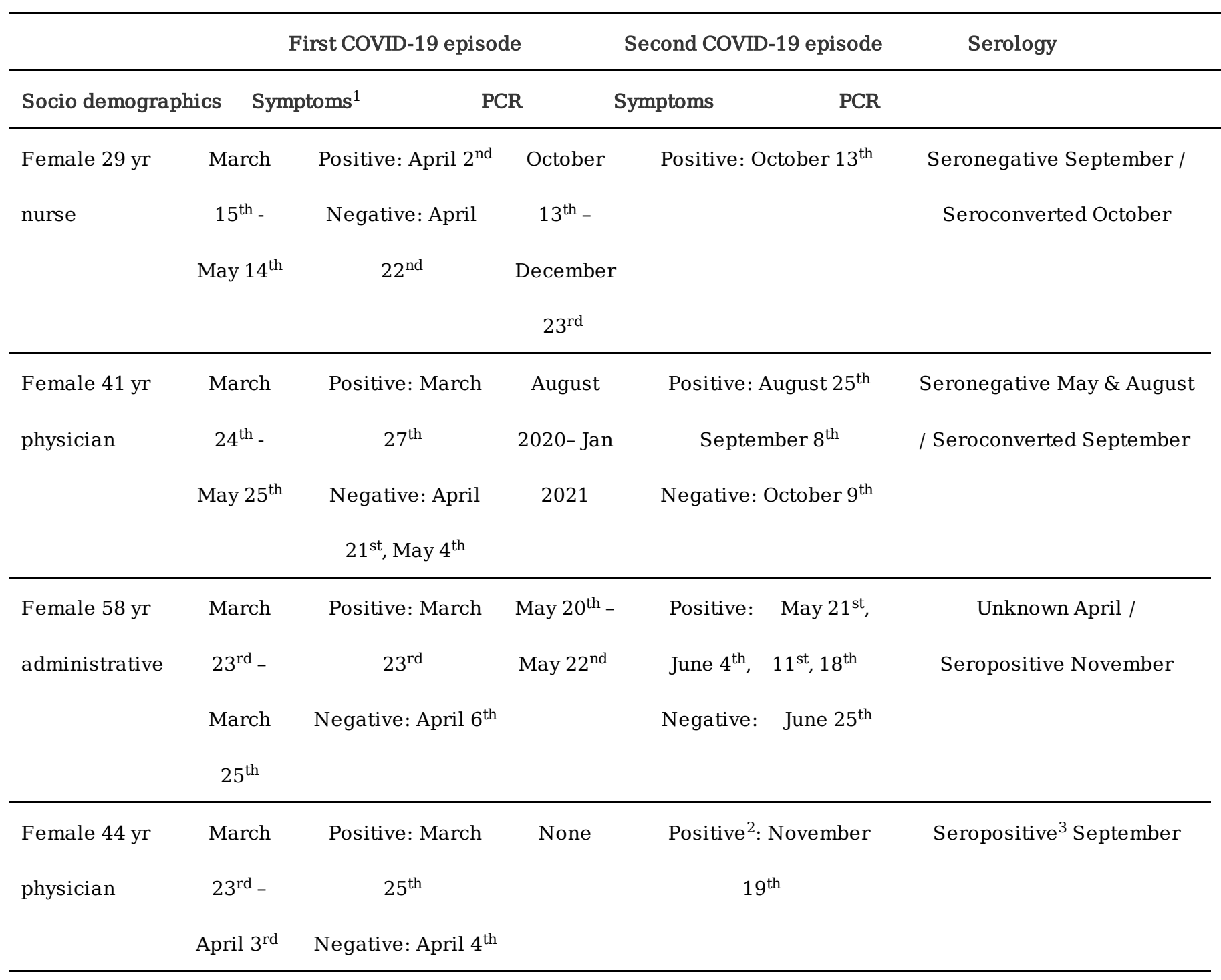

${ }^{1}$ Date of start and end of the first and last symptoms. All dates are 2020 unless otherwise indicated.

${ }^{2} \mathrm{PCR}$ was done prior to an unrelated surgical procedure and not as part of any routine COVID-19 screening, the participant had no symptoms.

${ }^{3}$ Low level antibody responses above the seropositivity threshold.

Table 2. Baseline variables associated with SARS-CoV-2 spike antibody levels 5-9 months after COVID-19 symptoms onset by multivariable stepwise regression models. 


\begin{tabular}{|c|c|c|c|c|c|c|c|c|c|}
\hline \multirow[b]{2}{*}{$\operatorname{IgM}^{2}$} & \multirow{2}{*}{$\begin{array}{l}\text { Predictors } \\
\text { Hospitalization }\end{array}$} & \multirow{2}{*}{$\frac{\text { Beta }^{1}}{0.187}$} & \multicolumn{2}{|c|}{$95 \% \mathrm{CI}^{3}$} & \multirow{2}{*}{$\frac{\mathrm{p} \text {-value }}{0.024}$} & \multirow{2}{*}{$\begin{array}{l}\text { Beta } \\
0.324\end{array}$} & \multicolumn{2}{|c|}{$95 \% \mathrm{CI}$} & \multirow{2}{*}{$\begin{array}{l}\text { p-value } \\
<0.001\end{array}$} \\
\hline & & & 0.025 & 0.348 & & & 0.150 & 0.498 & \\
\hline & Previous allergies & 0.157 & 0.000 & 0.314 & 0.051 & ns & ns & ns & ns \\
\hline & Sputum and/or hemoptysis & 0.156 & -0.050 & 0.363 & 0.137 & 0.268 & 0.047 & 0.489 & 0.018 \\
\hline & Anosmia/hypogeusia & 0.108 & -0.003 & 0.220 & 0.057 & 0.091 & -0.028 & 0.210 & 0.133 \\
\hline & Fever & 0.091 & -0.038 & 0.219 & 0.165 & 0.112 & -0.027 & 0.250 & 0.113 \\
\hline & Digestive alterations & ns & ns & ns & ns & -0.089 & -0.210 & 0.033 & 0.152 \\
\hline \multirow[t]{8}{*}{$\operatorname{Ig} \mathbf{A}$} & Fever & 0.250 & 0.094 & 0.406 & 0.002 & 0.178 & 0.060 & 0.296 & 0.003 \\
\hline & Previous allergies & $\mathrm{ns}^{4}$ & ns & ns & ns & 0.157 & 0.016 & 0.298 & 0.029 \\
\hline & Hospitalization & ns & ns & ns & ns & 0.156 & 0.013 & 0.299 & 0.033 \\
\hline & Shivers & 0.160 & 0.024 & 0.296 & 0.022 & 0.087 & -0.014 & 0.188 & 0.091 \\
\hline & Anosmia/hypogeusia & 0.139 & 0.004 & 0.273 & 0.043 & ns & ns & ns & ns \\
\hline & Smoking & -0.269 & -0.524 & -0.015 & 0.038 & -0.222 & -0.411 & -0.032 & 0.022 \\
\hline & Nurses & -0.265 & -0.443 & -0.086 & 0.004 & -0.223 & -0.357 & -0.090 & 0.001 \\
\hline & Physicians & -0.186 & -0.360 & -0.003 & 0.046 & -0.219 & -0.352 & -0.087 & 0.001 \\
\hline \multirow[t]{2}{*}{ IgG } & Anosmia/hypogeusia & 0.413 & 0.258 & 0.568 & $<0.001$ & 0.189 & 0.077 & 0.301 & 0.001 \\
\hline & Fever & 0.398 & 0.218 & 0.578 & $<0.001$ & 0.301 & 0.169 & 0.432 & $<0.001$ \\
\hline & Previous allergies & 0.269 & 0.053 & 0.485 & 0.015 & 0.137 & -0.021 & 0.295 & 0.090 \\
\hline & Hospitalization & 0.187 & -0.024 & 0.398 & 0.082 & 0.236 & 0.068 & 0.404 & 0.006 \\
\hline & Age & 0.007 & 0.000 & 0.014 & 0.050 & 0.006 & 0.001 & 0.011 & 0.023 \\
\hline & Cough & 0.124 & -0.034 & 0.283 & 0.123 & ns & ns & ns & ns \\
\hline & Digestive alterations & ns & ns & ns & ns & 0.088 & -0.025 & 0.202 & 0.126 \\
\hline & Smoking & -0.295 & -0.580 & -0.009 & 0.043 & ns & ns & ns & ns \\
\hline & Nurses & ns & ns & ns & ns & -0.187 & -0.335 & -0.039 & 0.014 \\
\hline & Physicians & ns & ns & ns & ns & -0.105 & -0.253 & 0.042 & 0.159 \\
\hline
\end{tabular}

${ }^{1}$ Estimate of the model (beta coefficient), see text for interpretation.

${ }^{2} \log _{10}$ MFI: logarithm 10 median fluorescent intensity (antibody levels).

${ }^{3} \mathrm{CI}$ : confidence interval of the model estimate (beta).

${ }^{4}$ ns: not significant (not retained in the stepwise forward/backward multivariable model) 
Figures
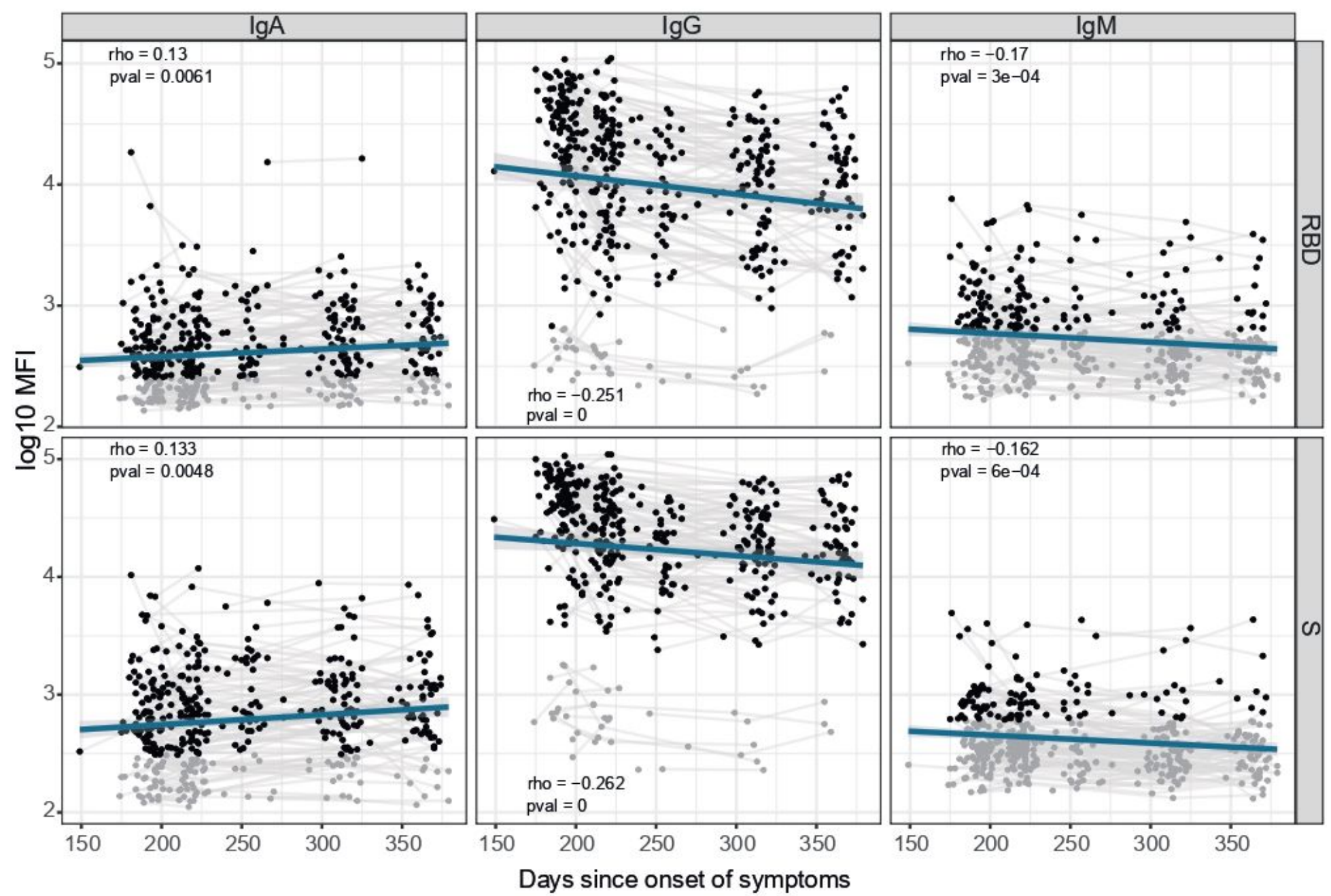

Result - Negative - Positive

Figure 1

SARS-CoV-2 antibody levels by days since COVID-19 symptoms onset. IgA, IgG and IgM levels are represented in log10 median fluorescence intensity (log10 MFI). Black dots represent seropositive individuals and grey dots represent seronegative individuals. Paired samples are joined by grey lines. The blue solid line represents the fitted curve calculated using the linear model method. Shaded areas represent $95 \%$ confidence intervals. 